\title{
MEJORAMIENTO DEL PROCESO DE DESHIDRATACIÓN DE UCHUVA
}

\author{
IMPROVING THE PROCESS OF \\ DEHYDRATION UCHUVA
}

\section{Rafael Giraldo, ${ }^{1}$ Tulio Vargas, ${ }^{2}$ Hildebrando Gil $^{3}$}

\begin{abstract}
RESUMEN
En el secado de alimentos es imprescindible conocer el tiempo necesario para conseguir la temperatura del bulbo seco, con el objetivo de reducir el consumo energético, sin alterar la calidad del producto. Se requiere una estimación adecuada para determinar previamente el coeficiente de transmisión de calor por convección forzada entre el aire y el producto. En este trabajo se desarrolló un procedimiento de un sistema de secado de la uchuva en forma semiesférica y en cuatro cascos en un deshidratador piloto de bandejas. El tiempo de calentamiento se determinó midiendo la pérdida de humedad vs el tiempo de secado. Con base en estadísticas reportadas por la empresa para la que se realizó la investigación, se seleccionaron como variables significativas las siguientes: temperatura de $58^{\circ} \mathrm{C}$, velocidad de entrada $3,55 \mathrm{~m} / \mathrm{s}$, presentación de dos cascos y temperatura de $62^{\circ} \mathrm{C}$, velocidad de 5,85 $\mathrm{m} / \mathrm{s}$ y presentación en cuatro cascos, respectivamente. Estos valores proporcionaron las combinaciones de las diferentes variables y se pudo concluir, mediante un diseño factorial 23 como condiciones favorables, la presentación en cuatro cascos, a una temperatura de bulbo seco de $62^{\circ} \mathrm{C}$ y velocidad de entrada de $3,55 \mathrm{~m} / \mathrm{s}$.
\end{abstract}

Con estos resultados se escalonaron las condiciones de secado mediante un análisis adimensional en el equipo industrial, manteniendo la temperatura de $62^{\circ} \mathrm{C}$ y la presentación de cuatro cascos, dando un resultado, para la velocidad de entrada de aire, de $8 \mathrm{~m} / \mathrm{s}$. Las condiciones mencionadas permitieron reducir el proceso de secado en 4 horas, con un porcentaje de humedad final de $13,8 \%$ sin alterar significativamente la calidad del producto.

Palabras clave: secado, transmisión de calor, humedad, convección.

\footnotetext{
ABSTRACT

In the drying of food is essential to know the time required to achieve the dry bulb temperature, with the goal of reducing energy consumption without affecting product quality. Appropriate

1 Especialista en Ingeniería de Procesos en Alimentos y Biomateriales. UNAD. E-mail: rgiraldot1@une.net.co.

2 Especialista en Ingeniería de Procesos en Alimentos y Biomateriales. UNAD. Gerente Empresa de Alimentos LE FRUIT, Medellín. E-mail: tuliop@une.net.co.

3 Especialista en Ingeniería de Procesos en Alimentos y Biomateriales. UNAD. Instructor de Alimentos y Farmacia, SENA Apartadó - Antioquia. E-mail: hildegil@hotmail.com.
} 
estimate is required to determine in advance the heat transfer coefficient by forced convection between air and product. In this paper we developed a procedure for a drying system in the form of Gooseberry hemispherical and four helmets in a pilot dehydrator trays. The heating time was determined by measuring the moisture loss vs. drying time. Based on statistics reported by the company which conducted the research, significant variables were selected as follows: temperature $58^{\circ} \mathrm{C}$, inlet velocity $3.55 \mathrm{~m} / \mathrm{s}$, presentation of two helmets and a temperature of $62^{\circ} \mathrm{C}$, velocity of $5.85 \mathrm{~m} / \mathrm{s}$ presentation at four feet, respectively. These values provided the combinations of different variables and it was concluded by a 23 factorial design as favorable conditions, the presentation in four hooves, a dry bulb temperature of $62^{\circ} \mathrm{C}$ and inlet velocity of $3.55 \mathrm{~m} / \mathrm{s}$.

With these results, the drying conditions scaled by a dimensionless analysis on industrial equipment, maintaining the temperature of $62^{\circ} \mathrm{C}$ and the presentation of four feet, giving a result for the air inlet velocity of $8 \mathrm{~m} / \mathrm{s}$. The above conditions allow to reduce the drying process in 4 hours, with a final moisture content of $13.8 \%$ without significantly affecting the quality of the product.

Key words: drying, heat transfer, humidity, convection.

Aceptado: abril 25 de 2009

Aprobado: mayo 26 de 2009

\section{INTRODUCCIÓN}

La producción de frutas y hortalizas frescas se ve afectada, tanto en su cantidad como en su calidad, por la degradación que sufre su acondicionamiento y por el transporte entre las zonas productoras y las áreas de consumo; asimismo, por un manejo inadecuado en transporte y almacenamiento, después de la recolección, incrementando así las pérdidas. El secado es una solución, no solo para disminuir costos en el transporte, sino para aumentar la vida útil del producto. El diseño adecuado de un sistema de secado convectivo forzado requiere conocer los fenómenos complejos de transferencia de calor y de masa. El desarrollo del trabajo experimental a nivel piloto con las variables planteadas, permitieron diseñar un modelo experimental factorial 23, ver (tabla 1).

Tabla 1. Variables y niveles

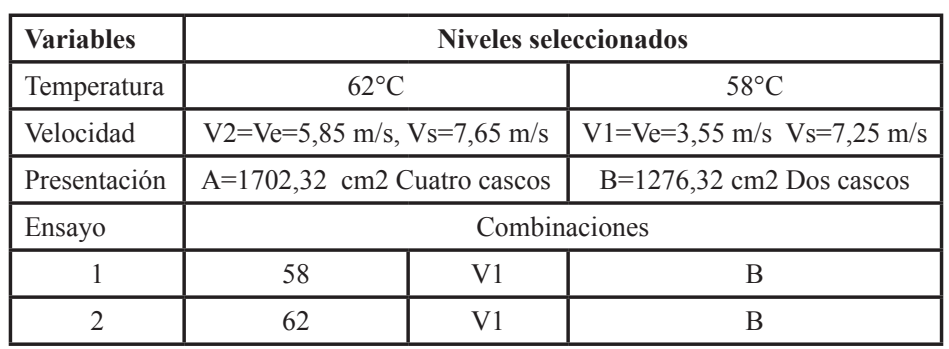




\begin{tabular}{|l|l|l|l|}
\hline 3 & 58 & V2 & B \\
\hline 4 & 62 & V2 & B \\
\hline 5 & 58 & V1 & A \\
\hline 6 & 62 & V1 & A \\
\hline 7 & 58 & V2 & A \\
\hline 8 & 62 & V2 & \\
\hline
\end{tabular}

Estas variables permitieron obtener resultados confiables de temperatura de bulbo seco, velocidad de entrada y forma del producto, mediante los cuales se determinaron los coeficientes de transferencia de calor a partir de correlaciones adecuadas. Además, fue posible conocer la solución analítica de la rata de transferencia de calor. El coeficiente de transferencia de calor se calculó con base en el análisis dimensional, teniendo en cuenta los números adimensionales Nusselt, Reynolds y Prandtl. De esta forma se determinó la velocidad de entrada en un equipo en el sector industrial partiendo de los resultados del modelo piloto. [12]

Existe una relación genérica entre los grupos adimensionales en un sistema de secado convectivo:

$$
N u=f(\operatorname{Re}, \operatorname{Pr})
$$

Donde :

$N u=\frac{h L c}{k}, \operatorname{Re}=\frac{v \rho L c}{\mu} ; \operatorname{Pr}=\frac{c_{p} \mu}{k}$

Siendo v(ms $\left.{ }^{-1}\right), \mu\left(\mathrm{Kgm}^{-1} \mathrm{~s}^{-1}\right)$ y $\mathrm{Lc}(\mathrm{m})$ la velocidad, la viscosidad absoluta del aire y la longitud característica correspondiente a la longitud de la bandeja, respectivamente.

El $\mathrm{Cp}\left(\mathrm{JKg}^{-1} \mathrm{~K}^{-1}\right)$, el calor específico; $\mathrm{k}\left(\mathrm{Wm}^{-1} \mathrm{~K}^{-1}\right)$, la conductividad térmica del aire $\mathrm{y}$, por último, $\mathrm{h}\left(\mathrm{Wm}^{-2} \mathrm{~K}^{-1}\right)$, el coeficiente de transferencia de calor por convección forzada, permiten la solución analítica para la transferencia de calor y son el punto de partida para hacer el escalonamiento a nivel industrial [1], [11]

Durante el período de velocidad del secado constante la humedad que se remueve del producto es aquella que se encuentra en su superficie; la remoción es similar a la evaporación del agua desde una superficie libre. La velocidad de secado depende, principalmente, de las características del ambiente que rodean al producto y, en menor grado, de la naturaleza del material. El período termina en un punto conocido como humedad crítica y se presenta cuando en el producto la velocidad de difusión de la humedad en su interior decrece más allá de la cantidad de humedad necesaria para ser movida por convección desde la superficie [7], [10].

Los fenómenos de transporte que tienen lugar son la transferencia de masa de vapor de agua al ambiente desde la superficie del producto y la transferencia de calor a través del sólido. Durante el 
proceso de secado, la superficie del producto permanece saturada de agua, ya que la velocidad del movimiento del agua desde el interior del sólido es suficiente para compensar el agua evaporada en la superficie. Si solo se presentan transferencia de calor por convección (despreciando la radiación desde el aire caliente a la superficie del sólido) y transferencia de masa desde la superficie al aire caliente, los balances de masa y energía se pueden expresar como [8]:

$$
\begin{aligned}
& q=h A\left(T-T_{W}\right) \\
& N_{a}=K_{y}\left(Y_{w}-Y\right)
\end{aligned}
$$

$\mathrm{h}$ es el coeficiente de transferencia de calor; $\mathrm{A}$, el área de secado; $\mathrm{T}_{\mathrm{W}}$ es la temperatura de bulbo húmedo; T es la temperatura de secado; $\mathrm{Na}$, la densidad de difusión; Yw, el contenido de humedad del aire en la superficie del sólido; Y, el contenido de humedad del aire en el seno de la corriente de secado y $\mathrm{K}_{\mathrm{Y}}$, el coeficiente de transferencia de masa [2].

El calor necesario para vaporizar el agua en la superficie del producto se puede expresar como:

$q=N_{a} M_{a} \lambda_{w} A$

Ma es la masa molecular del agua y $\lambda_{\mathrm{w}}$ es el calor latente de vaporización a la temperatura $\mathrm{T}_{\mathrm{w}}$. La velocidad de secado en el producto se puede expresar según ecuación (Okos et al, 1992):

$$
\begin{aligned}
& R_{c}=K_{y} M_{b}\left(H_{w}-H\right) \\
& R_{c}=\frac{h\left(T-T_{w}\right)}{\lambda_{w}}=\frac{q}{\lambda_{w} A}
\end{aligned}
$$

$\mathrm{M}_{\mathrm{b}}$ es la masa molecular del aire; $\mathrm{H}_{\mathrm{w}}$, la humedad correspondiente a la temperatura de bulbo húmedo y $\mathrm{H}$ es la humedad del aire en la corriente gaseosa.

El coeficiente de transmisión de calor se puede expresar en una lámina en función del módulo de Nusselt, según la siguiente expresión:

$$
\begin{aligned}
& N u=\frac{h d}{k}=0.664 \operatorname{Re}^{0.5} \operatorname{Pr}^{1 / 3} ; \operatorname{Re}<500.000 \\
& N u=\frac{h d}{k}=0.0366 \operatorname{Re}^{0.8} \operatorname{Pr}^{1 / 3} ; \operatorname{Re}>500.000
\end{aligned}
$$

Las ecuaciones (5) y (6) son útiles para determinar la velocidad de secado en el periodo de velocidad constante. No obstante, es preferible realizar mediciones experimentales. 
El tiempo de secado en este periodo de velocidad constante se calcula resolviendo la ecuación:

$$
\begin{aligned}
& R=-\frac{F_{s}}{A}\left(\frac{d X}{d t}\right)(9) \quad t=\int_{t_{1}=0}^{t_{2}} d t=-\frac{F_{s}}{A} \int_{X_{1}}^{X_{2}} \frac{d X}{R} \\
& t_{c}=\frac{F_{s} \lambda_{w}\left(X_{1}-X_{2}\right)}{A h\left(T-T_{w}\right)}=\frac{F_{s}\left(X_{1}-X_{2}\right)}{A K_{y} M_{b}\left(H_{w}-H\right)}
\end{aligned}
$$

\section{MATERIALES Y MÉTODOS}

\section{Secador de bandejas}

En el siguiente diagrama se muestra el procedimiento experimental realizado con el producto o las diferentes muestras

\section{DIAGRAMA DE FLUJO}

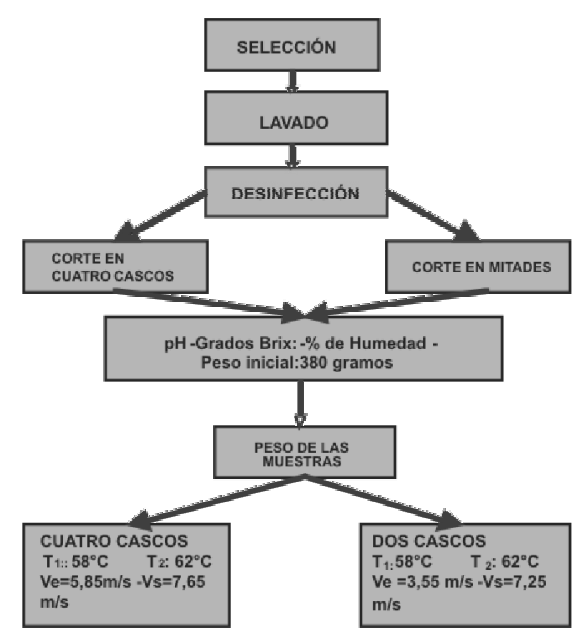

De un bulto de 40 kilos, se tomó una muestra al azar de 380 gramos, pesada en una balanza analítica; con un pie de rey se midió el diámetro de cada una de las uchuvas, cuyo promedio fue de $21,25 \mathrm{~mm}$. Con este diámetro se calculó el área superficial de las dos presentaciones, asumiendo una geometría esférica. Las áreas calculadas fueron: $1276,74 \mathrm{~cm} 2$ y 1702,32 cm².

Se determinó el contenido de humedad mediante estufa de circulación de aire caliente a $105^{\circ} \mathrm{C}$ hasta peso constante, el cual fue equivalente al $79,1 \%$. 
El producto se llevó a un secador piloto de 9 bandejas de $40 \mathrm{~cm} \mathrm{x} 40 \mathrm{~cm}$, de tipo armario por convección forzada, cuya fuente de calor proviene de resistencias eléctricas ubicadas en la parte lateral y un ventilador situado en la parte posterior el cual permite una circulación de aire paralelo a las placas. Para cada ensayo se midieron las velocidades con un anemómetro con el fin de garantizar los valores del modelo planteado. Al cabo de diez minutos, una vez estabilizada las condiciones, se midieron las temperaturas de bulbo seco y la del medio ambiente con termómetro de carátula. Cada diez minutos y por medio de una balanza electrónica de precisión se pesaba la muestra y se repetía el procedimiento hasta alcanzar un tiempo de 300 minutos.

Los datos y los gráficos se han obtenido en entorno Windows mediante Excel 2003 y los software Curve expert 3.2, Matlab 6.5, Heatxfer, Statgraphics plus versión [5.1].

\section{Periodo de velocidad constante y transferencia de calor}

\section{- Periodo de velocidad constante}

En el periodo donde se elimina la mayor cantidad de agua, correspondiente al periodo de velocidad constante, se determinaron las velocidades de secado mediante las ecuaciones:

$$
R=-\frac{F_{s}}{A}\left(\frac{\Delta X}{\Delta t}\right) \text { y } x_{T}=\left(\frac{w_{T}-F_{S}}{F_{s}}\right),
$$

Donde Fs $\left(\mathrm{Kg} \mathrm{s}^{-1}\right)$ son los sólidos secos en el producto; $\mathrm{A}\left(\mathrm{m}^{2}\right)$, área superficial total de la muestra; $\Delta \mathrm{X}\left(\mathrm{Kg} \mathrm{H} 2 \mathrm{O} / \mathrm{Kgs}^{-1} \mathrm{~s}^{-1}\right)$, la diferencia de humedades en un periodo de $\Delta \mathrm{t}$ de $0.1666 \mathrm{hr}$ correspondiente a 10 minutos; $\mathrm{W}_{\mathrm{T}}(\mathrm{Kg})$, peso total de la muestra y $\mathrm{X}_{\mathrm{T}}\left(\mathrm{KgH} 2 \mathrm{O} / \mathrm{Kgs} \mathrm{s}^{-1}\right)$, humedad en base seca.

\section{Transferencia de calor por convección}

El cálculo de los grupos adimensionales, $\mathrm{Re}, \mathrm{Nu}$ y $\mathrm{Pr}$ relacionados con la transferencia de calor por convección forzada, se realizó a partir de sus definiciones y las propiedades del aire de secado al promedio de temperatura de bulbo seco y húmedo, mediante la ayuda del software HeatXfer y Carta Psicométrica [5].

\section{Análisis de resultados}

Las tablas 2 y 3 muestran los resultados experimentales de los ensayos 1, 2, 3, 4, 5, 6, 7 y 8 para las condiciones de velocidad de entrada, temperatura de bulbo seco y las dos presentaciones.

Las gráficas 1, 2, 3 y 4 muestran las curvas correspondientes a los ensayos 1, 2, 3, 4, 5, 6, 7 y 8 representados en humedad en base seca vs tiempo de secado y las ratas de velocidad de secado de los ensayos. 
Tabla 2. Resultados de los ensayos 1-2-3-4

\begin{tabular}{|l|r|r|r|r|r|}
\hline & $\begin{array}{c}\text { TIEMPO } \\
\text { CRITICO } \\
\text { (min) }\end{array}$ & $\begin{array}{c}\text { AGUA } \\
\text { ELIMINADA } \\
\text { HASTA Xc (gr) }\end{array}$ & \multicolumn{1}{c|}{ Xc } & $\begin{array}{c}\text { AGUA ELIMINADA } \\
\text { (gr) DESDE Xc } \\
\text { HASTA 300 min }\end{array}$ & X a 300 min \\
\hline ENSAYO 1 & 160 & 139.93 & 2.02 & $15 \mathrm{~g}$ & 9.75 \\
\hline ENSAYO 2 & 200 & 220.95 & 1.0026 & 22 & 0.8799 \\
\hline ENSAYO 3 & 180 & 124.73 & 2.2142 & 9.92 & 2.1790 \\
\hline ENSAYO 4 & 180 & 173.02 & 1.6062 & 1.4812 \\
\hline
\end{tabular}

Gráfico 1. Humedad en base seca vs tiempo

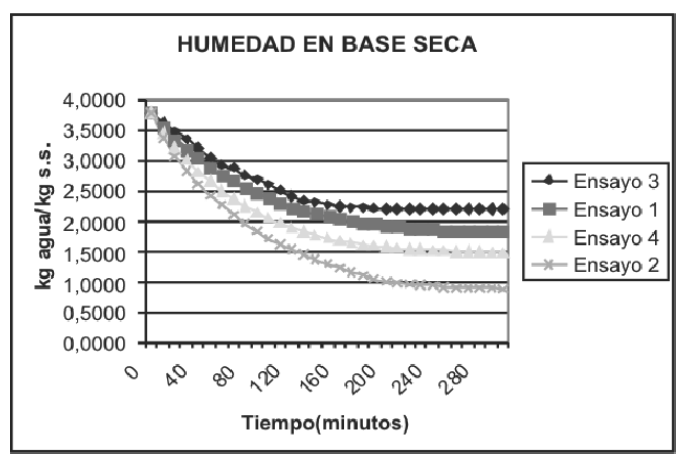

Gráfico 2. Curvas de velocidad de secado

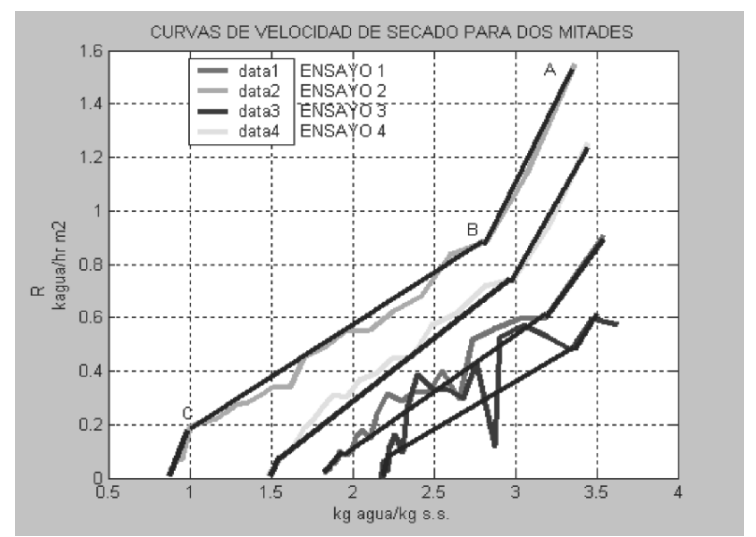


Tabla 3. Resultados de los ensayos 5-6-7-8

\begin{tabular}{|l|r|r|r|r|r|}
\hline & $\begin{array}{c}\text { TIEMPO } \\
\text { CRITICO } \\
(\mathbf{m i n})\end{array}$ & $\begin{array}{c}\text { AGUA } \\
\text { ELIMINADA } \\
\text { HASTA Xc }(\mathbf{g r})\end{array}$ & $\mathbf{X c}$ & $\begin{array}{c}\text { Agua eliminada } \\
\text { (gr)desde Xc hasta } \\
\mathbf{3 0 0} \mathbf{~ m i n}\end{array}$ & X a 300 min \\
\hline ENSAYO 5 & 250 & 178.77 & 1.5337 & 5.11 & 1.4694 \\
\hline ENSAYO 6 & 250 & 276.39 & 0.3046 & 2.07 & 0.2785 \\
\hline ENSAYO 7 & 200 & 152.99 & 1.8583 & 4.66 & 1.7997 \\
\hline ENSAYO 8 & 200 & 205.33 & 1.1993 & 4.85 & 1.1383 \\
\hline
\end{tabular}

Grafico 3. Humedad en base seca de los ensayos 5-6-7-8 para la presentación en cuatro cascos

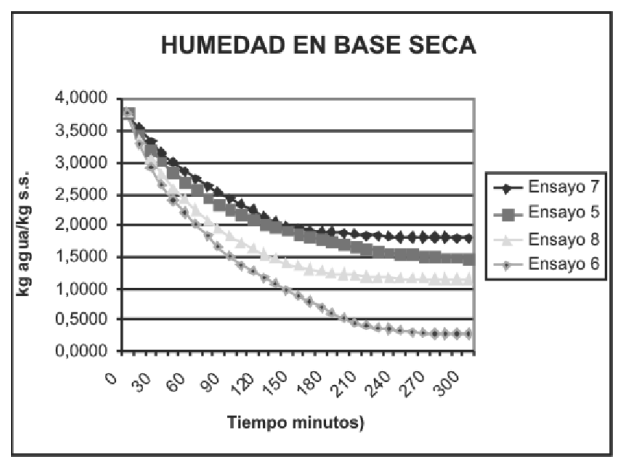

Gráfico 4. Humedad en base seca de los ensayos 5-6-7-8 para la presentación en cuatro cascos

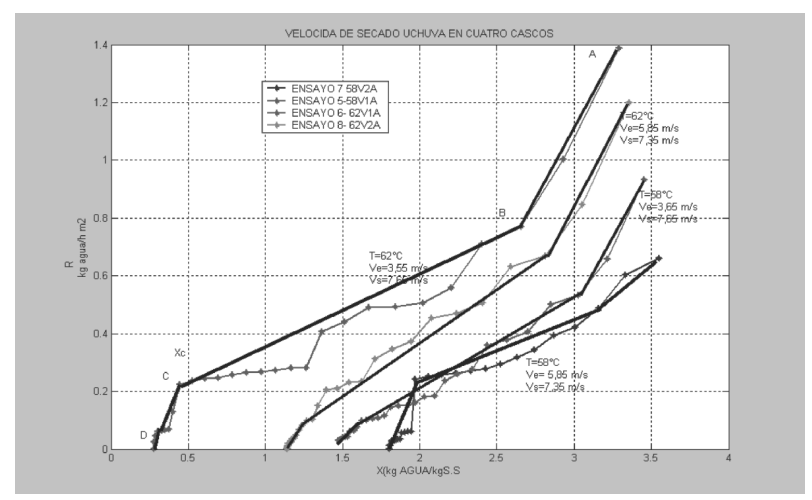

En las gráficas 5 y 6 aparecen las curvas de humedad en base seca y rata de velocidad de secado de los dos mejores ensayos, correspondientes a las dos presentaciones. 
Gráfico 5. Comparación de los ensayos 2 y 6 en humedad en base seca

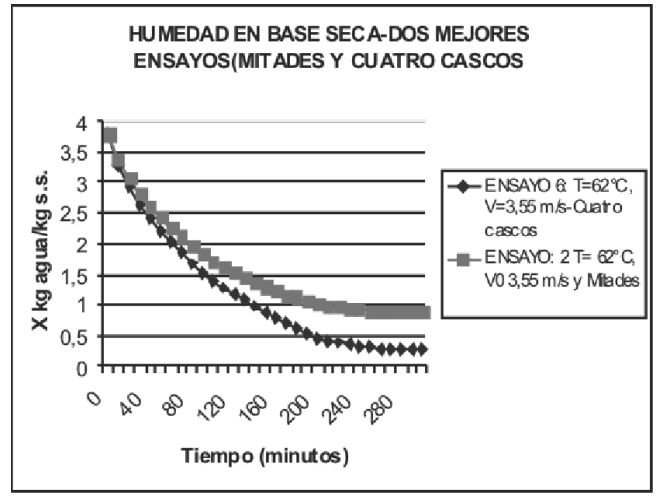

Gráfico 6. Comparación de los ensayos 2 y 6

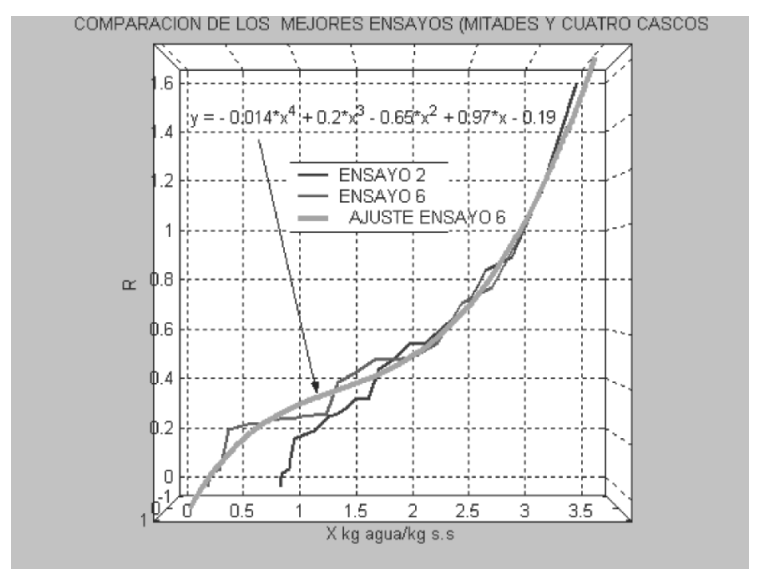

Gráfico 7. Rata de velocidad de secado ensayo 9

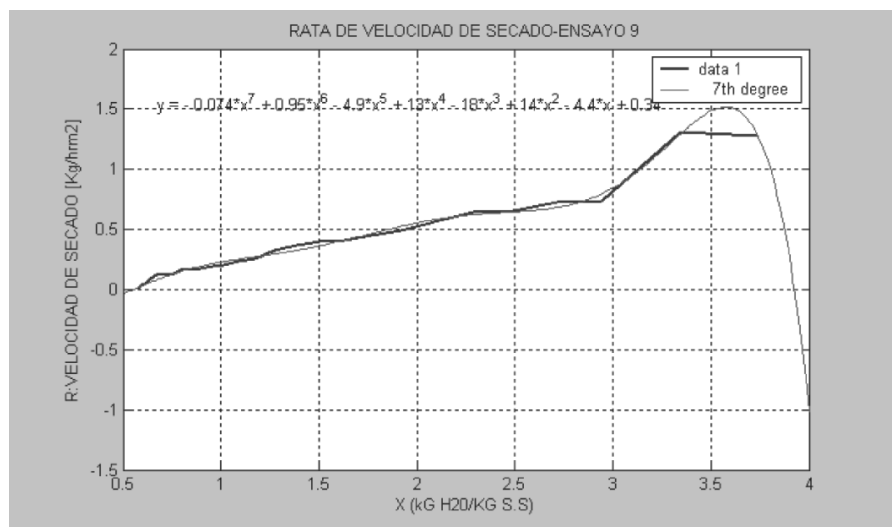




\section{ANÁLISIS DE RESULTADOS}

En estas gráficas se representa la velocidad de secado R vs humedad en base seca X, con el fin de visualizar las diferentes etapas del periodo de secado. En el ensayo 2 de la presentación de mitades, en el que se presentan las condiciones más favorables de los cuatro primeros ensayos, el tramo de la curva A-B corresponde a la eliminación de agua desde la superficie, la cual se encuentra muy húmeda y empieza su periodo de calentamiento hasta llegar a la temperatura de bulbo húmedo correspondiente al punto $\mathrm{B}$, donde se inicia el periodo de velocidad constante. En el tramo B-C del periodo de velocidad constante se elimina el agua libre donde la actividad del agua es cercana a la unidad [6]. Continúa este periodo, mientras el agua evaporada en la superficie es reemplazada por la proveniente del interior. En este periodo, las condiciones dependen de los factores externos de temperatura, humedad y velocidad de aire. La velocidad del aire seco corresponde a $3,55 \mathrm{~m} / \mathrm{s}$ y permite mayor tiempo de residencia, así como el retirar mayor cantidad de agua, ya que hay un gradiente más alto de temperatura, el cual debe llegar a un límite que no permita el encogimiento de la superficie. El periodo de velocidad constante termina en $\mathrm{C}$, iniciándose el periodo de velocidad decreciente, donde la actividad del agua se hace menor.

Para los ensayos 5, 6, 7 y 8, el seis presenta las condiciones más aceptables. El tramo de la curva A-B corresponde a la eliminación de agua desde la superficie, la cual se encuentra muy húmeda, y empieza su periodo de calentamiento hasta llegar a la temperatura de bulbo húmedo correspondiente al punto $\mathrm{B}$, momento en el que se inicia el periodo de velocidad constante.

En el tramo B-C del periodo de velocidad constante se elimina el agua libre, donde la actividad del agua es cercana a la unidad. Continúa este periodo, mientras el agua evaporada en la superficie es reemplazada por la proveniente del interior; la velocidad de secado depende de los factores externos de temperatura, humedad y velocidad de aire. En este caso, la velocidad del aire seco es de $3,55 \mathrm{~m} / \mathrm{s}$ y permite mayor tiempo de residencia; de la misma forma, se puede retirar mayor cantidad de agua, puesto que hay un gradiente más alto de temperatura, el cual debe llegar a un límite que no permita el agrietamiento en la superficie.

De acuerdo con los resultados del modelo experimental, el ensayo 6 presentó la mejor combinación para el proceso. Los datos de velocidad de entrada, $3.55 \mathrm{~m} / \mathrm{s}$, y la temperatura de $62^{\circ} \mathrm{C}$ para la presentación de cuatro cascos son el punto de partida para definir los valores de las variables en el secador de bandejas de la empresa.

Para el escalonamiento, la temperatura del aire de secado y la presentación se dejaron constantes de acuerdo con el ensayo 6. La velocidad resultante para el modelo industrial, calculada con el análisis dimensional, fue de $8 \mathrm{~m} / \mathrm{s}$. Estas condiciones mejoraron notablemente las ratas de velocidad de secado, disminuyendo el tiempo en cuatro horas. 


\section{CONCLUSIONES}

Los ensayos realizados con la presentación de mitades presentaron velocidades de secado menores a la presentación en cuatro cascos, debido a la menor área de contacto superficial.

Las velocidades de $3,55 \mathrm{~m} / \mathrm{s}$ permitieron mayores tiempos de residencia en las muestras, lo que facilita mayor extracción de agua.

Las temperaturas de $62^{\circ} \mathrm{C}$ combinadas con las velocidades de $3,55 \mathrm{~m} / \mathrm{s}$ presentaron los mejores resultados en el equipo piloto, lo que indica que en la superficie no se formaron películas secas que impidieran el paso de la humedad hacia el exterior. La compensación entre un gradiente de temperatura alta con una baja velocidad, genera un coeficiente convectivo más bajo debido a la disminución del $\mathrm{N}^{\circ}$ Reynolds, lo que influyó en las condiciones de mejora del proceso.

Las velocidades de $5,85 \mathrm{~m} / \mathrm{s}$ combinadas con las temperaturas de $62^{\circ} \mathrm{C}$ en el equipo piloto presentaron grandes dificultades en la velocidad de secado, ya que rápidamente se llegaba al punto crítico, quizás por la formación de una película impermeable que impedía el paso del agua.

De acuerdo con la parte estadística, las tres variables (temperatura, velocidad y área superficial) son significativas en el proceso de secado, con respecto a la humedad en base seca.

Con el fin de verificar mejoras en el proceso de secado en el deshidratador de bandejas de la Empresa, se realizó una deshidratación de la uchuva con los resultados del mejor ensayo (ensayo 6).

El escalonamiento de equipo piloto a equipo industrial con las condiciones de temperatura de $62^{\circ} \mathrm{C}$ y presentación de cuatro cascos de acuerdo con el ensayo 6 registró una velocidad de $7,987 \mathrm{~m} / \mathrm{s}$, teniendo en cuenta los grupos adimensionales Reynolds y Nusselt.

El tiempo de secado en el equipo industrial se redujo en 4 horas, lo que representa un beneficio económico para la Empresa

El tiempo teórico de 97,79 minutos y la rata de velocidad de secado teórica de $0,560468 \mathrm{Kg} /$ hrm 2 para el ensayo 6 no presentaron desviaciones muy marcadas con los datos experimentales de 150 minutos y $0.3978 \mathrm{Kg} / \mathrm{hrm} 2$ en el periodo de velocidad constante. Estas diferencias se presentan por el cambio del área superficial del producto, debido a su morfología.

El ensayo 9 correspondiente a la media de los niveles alto y bajo $\mathrm{T}=60^{\circ} \mathrm{C}, \mathrm{V}=4.70 \mathrm{~m} / \mathrm{s}$ y presentación de 3 cascos, permitió calcular el error experimental con respecto al ensayo 6 , dando como resultado una desviación estándar de 0,0168 , lo que genera confiabilidad en los valores de este ensayo. 
Al producto obtenido se le efectuaron las pruebas microbiológicas, las cuales arrojaron resultados positivos con los límites aceptables, según Invima.

Análisis del producto correspondiente al ensayo 6

- Características sensoriales

Color: amarillo

Olor: característico

Aspecto: fruta deshidratada, libre de partículas extrañas

\section{- Parámetros microbiológicos}

Rto de Mesófilos ufc/g 40 Límite permisible Invima $<10000$

NMP de Coniformes $/ g<3$ Límite permisible Invima $<3$

NMP de Coniformes fecales/g $<3$ Límite permisible Invima $<3$

Análisis fisicoquímico:

Humedad, 13.83\% Método Icontec GTC 1(1-14-2)

Las propiedades organolépticas no sufrieron cambios significativos en cuanto a sabor, color y textura, similares a las del producto que la empresa ha comercializado.

\section{REFERENCIAS BIBLIOGRÁFICAS}

[1] BARBOSA, Gustavo V, VEGA MERCADO, Humberto. Deshidratación de Alimentos. 2000 Editorial Acribia Zaragoza (España).

[2] BRENNAN,J.G., BUTTERS,J.R,COWELLl,N.D., y LILLEY, A.E.U.. En Advances inDrying, vol4, Editado por A.S.Mujundar. Hemisphere Publishing, New York.

[3] GEANKOPOLYS, C.J. Drying of Process Materials. En Transport Processes and Unit Operations, 3a edition. 2000.Allyn and Bacon, Boston, MA.

[4] HOLMAN, J. P. Experimental Methods for Engineers, 5a. Edition, MaGraw -Hill, New York, 1989.

[5] INCROPERA, F. C, y DeWITT,D.P. Introduction to Heat Transfer, 3a edition., John Wiley Sons. New York 1990.

[6] KUELH, Robert O. Diseño de experimentos. Principios estadísticos para el diseño y análisis de investigaciones $2^{\mathrm{a}}$ editcion México. 
[7] Mc CABE. WARREN L.,SMITH. Julian C.,HARRIOT. Meter. Operaciones Unitarias en Ingeniería Química. 2002 Mc Graw-Hill/Interamericana Editores, S.A, de C.V.

[8] MEJIA RUIZ, Carlos Alberto, ZULUAGA GIRALDO,. Victor Hugo. Trabajo de Grado "Selección de un Proceso para Deshidratar Uchuva (Physallis Peruviana). Escuela de Ingeniería, Departamento de Ingenierá de Procesos.2003 Universidad EAFIT. Medellín.

[9] MILLS,A. F.,Transferencia de Calor. 1995, Editorial Irwin España.

[10] SHRI K. SHARMA, Steven.J MULVANEY,Syed S.H.Rizvi. Ingenieria de Alimentos, 2003 Editorial Limusa, S.A.D Mexico.

[11] SMITH,P.G. Introduction To Food Process Engineering. Editorial Board.Kluwer Academic/Plenum Publisher. 2002 New York, Boston, Dordrecht, Moscow,

[12] PERFIL DE PRODUCTO N¹3. Inteligencia de Mercados. Uchuva. Pag 1-13.. editor Corporación Colombia Internacional. Bogotá Colombia 